Research Article

\title{
Advances in an Event-Based Spatiotemporal Data Modeling
}

\author{
Xinming Zhu, Haiyan Liu, Qing Xu (D), Jun'nan Liu, and Xiaoyang Lihua \\ Institute of Surveying and Mapping, Information Engineering University, Zhengzhou 450000, Henan, China
}

Correspondence should be addressed to Qing Xu; geozz_xd@whu.edu.cn

Received 4 October 2021; Revised 28 October 2021; Accepted 1 November 2021; Published 14 December 2021

Academic Editor: Ahmed Farouk

Copyright ( 2021 Xinming Zhu et al. This is an open access article distributed under the Creative Commons Attribution License, which permits unrestricted use, distribution, and reproduction in any medium, provided the original work is properly cited.

Spatiotemporal data are vitally important for the national economy and defense modernization since it is not only an important component of human society and geographical information of the environment but also a key carrier of spatiotemporal information. An event-based spatiotemporal data model and its improvements are employed to model spatiotemporal objects, change history, and change relation, which is the main approach to resolve the spatiotemporal change modeling and has been comprehensively developed in modeling theory and applications. This manuscript studies the event-based spatiotemporal data modeling theory based on three aspects of the cognitive theory, which are the spatiotemporal object, the concept of the spatiotemporal dynamic object, and the spatiotemporal object relationship. Then, the implementation characteristics of the models were analyzed regarding the management of cadastral information, analog natural disaster phenomena, and reasoning. Finally, the key points and difficulties of an event-based spatiotemporal data modeling and prospective developmental trends were discussed to provide insights with spatiotemporal data modeling.

\section{Introduction}

With the increasing implementations of a series of new geospatial technologies, such as Mobile Internet and Internet of Things (IoT), the acquisition cycle of spatial information becomes much shorter and the amount and coverage of spatiotemporal data keep on increasing and expanding, including the appearance of typical spatiotemporal data such as trajectory and environmental detection.

Abundant spatiotemporal data are the basis for the expression of spatiotemporal phenomena and knowledge mining as they contain information about the occurrence and evolution of spatiotemporal phenomena [1]. They are of great significance to the national economy and defense modernization. The research of spatiotemporal data model has gone through three major periods [2]: (1) the temporal snapshot period focusing on recording changes in entity state; (2) the object change period focusing on the expression of the relationship before and after an entity change, and (3) the event and activity period focusing on the description of semantic relations of an entity change.

The event-based spatiotemporal data model (ESTDM) and its improvements cope with the typical representatives of the third period. By modeling spatiotemporal objects, spatiotemporal changes, and relations of a change, it acquires the characteristics of explicitly modeling causes, processes, and results of spatiotemporal changes [3]. It has unique advantages that include the formation and evolution simulations of spatiotemporal phenomena, the knowledge mining of spatiotemporal changes, and discoveries of potential historical processes of the Earth $[4,5]$.

However, the further improvements and applications of the model are restricted since the ESTDM and its improvements have been mainly employed at specific scientific problems and applications. This research comprehensively considered the basic process of spatiotemporal data modeling, reviewed the theoretical basis and application characteristics of the existing models, analyzed the theoretical research status of the cognitive theory of spatiotemporal object, spatiotemporal dynamic object concept, and spatiotemporal object relationship, and summarized the application characteristics of the model in cadastral management and both re-enactment and reasoning of a physical geographic phenomenon, which is expected to provide a reference with an improvement and application of the ESTDM. 
The rest of the manuscript is organized as follows: Section 2 briefly introduces the theoretical research regarding the ESTDM based on the cognitive theory and its concepts. The research coverage related to implementation areas is presented in Section 3. Section 4 presents the effectiveness of the ESTDM model when spatiotemporal information, changes, and object relations exist. Besides, the issues are underlined. Section 5 concludes the research.

\section{Theoretical Research on the ESTDM}

Peuquet [6] proposed the ESTDM in 1995, which deals with the change of each state such as shape and attribute of the spatial region as an event, organizes events in temporal order, and expresses a process of a discrete spatiotemporal change of the spatial region from the initial state to the final change. In the real world, the relationships between entity and environment as well as interentity alter constantly. Aiming at dealing with the problem of change expression, researchers have proposed a variety of theories and methods that improve and apply the model in the realm of problems that could probably appear in the future. It is necessary to sort out and standardize the concepts of events, processes, states, and spatial regions and re-establish the concepts and relations of various elements of the ESTDM by utilizing the available achievements of the cognitive theory of a spatiotemporal object.

\subsection{The Cognitive Theory of Spatiotemporal Object.} Spatiotemporal objects are entities and changes that existed in spatiotemporal dynamic phenomena. They can be comprehensively recognized based on the way that they exist in the time-space domain and the characteristics of changes that they undergo. Theories analyzing the characteristics of object existence in space and time domains include both endurantism and perdurantism [7-9]. While the object of endurantism is only expanded in the space domain and a snapshot at any time is its complete form, the object of perdurantism is expanded in both time and space domains, and "change" is the uneven distribution of an object in the time domain. There are continuum and contingency theories to analyze the characteristics of objects in terms of experiencing change [10]: continuum is considered a change without an alteration in nature and can be used to describe relatively stable geographical objects, such as people, the Earth, and other geographical entities. On the other hand, contingency occurs in the time domain, having clear time boundaries, acting on the continuums, and expressing the history of the continuums.

To sum up, spatiotemporal objects can be divided into geographic objects that can change, have spatial attributes and any snapshots as a complete existence, and are dynamic objects with changes embedded in the interior and strong temporal attributes. Meanwhile, spatiotemporal objects are independent, nonseparable, and occupy a certain range of space, that is, they have properties called independence, atomicity, and scope. The characteristics of spatiotemporal objects are shown in Table 1.
2.2. The Concept of a Spatiotemporal Dynamic Object. Spatiotemporal dynamic objects are the carriers of dynamically changing semantics and events. Processes are common spatiotemporal dynamic objects. Different improved models embrace different fields and scientific problems, and the concepts of events and processes get confused and the boundaries are not clear [11]. The ESTDM puts forward the concept of an event in the field of geographic information and expresses the significant change with clearer endpoints for the first time. The events of spatiotemporal dynamic objects in this manuscript follow this concept. For the concept of a process, the characteristics, representation of a structured process, and homogenization process based on Galton's research [12] are discussed in this manuscript.

2.2.1. The Structured Process. A structural process refers to the spatiotemporal evolution process consisting of subevents organized in a certain structure and having a definite endpoint (but not yet arrived). For example, the evolution process of the ESTDM is represented by an event sequence organized in a temporal order. The representation methods of a structured process can be divided into the representation methods based on logical relations and based on temporal hierarchy when the temporal order and the different internal event organization methods are a concern.

(1) The method of the process representation based on a logical relationship

The process representation method based on logical relationship records the state change of the object, event causality, object evolution relationship, and the relationship of the event-process interaction concerning a temporal order. The changed semantics expressed by the model is richer. The model designed based on this method is shown in Table 2 .

Zhang et al. [13] designed a process-based spatiotemporal data model (PBSTDM), which combines logically connected events obtained by both classification and abstraction with the object state to form a spatiotemporal change process. Then, it depicts the relationship of the parent-child change of the object at the same time by expressing the semantics of the change process. The spatiotemporal data model based on the graph theory [17] employs the causal evolution process of the state-event-state situation expressed by Petri net place, transition, and directed arc [14], which effectively expresses the temporal prepost relationships and the successful relationships such as element replacement, merging, and segmentation. Chen et al. $[15,16]$ proposed that process ontology can be composed of object and event ontologies and is multiple event ontologies occurring in one (multiple) object ontology in a continuous time. The process ontology can also be composed of the process ontologies. Object ontology has the participation relations of generation, support, and 
TABLE 1: Analyzing the characteristics of spatiotemporal objects.

\begin{tabular}{|c|c|c|}
\hline & Geographic object & Dynamic object \\
\hline Similarities & Aton & $\begin{array}{l}\text { are independent of each other } \\
\text { object are not of the same type as itself } \\
\text { ains, while dynamic objects extend in the time domain }\end{array}$ \\
\hline Diff & $\begin{array}{l}\text { Can go through changes } \\
\text { Has spatial properties } \\
\text { But no temporal properties exists intact at any point in its life cycle }\end{array}$ & $\begin{array}{c}\text { Have changes embedded within the object } \\
\text { Has a temporal part } \\
\text { Cannot be expressed completely for a while in its life cycle }\end{array}$ \\
\hline
\end{tabular}

TABLE 2: Description of a process representation model based on a logical relationship.

\begin{tabular}{llc}
\hline Model diagram & & Model characteristics \\
\hline Start & Process & End
\end{tabular}

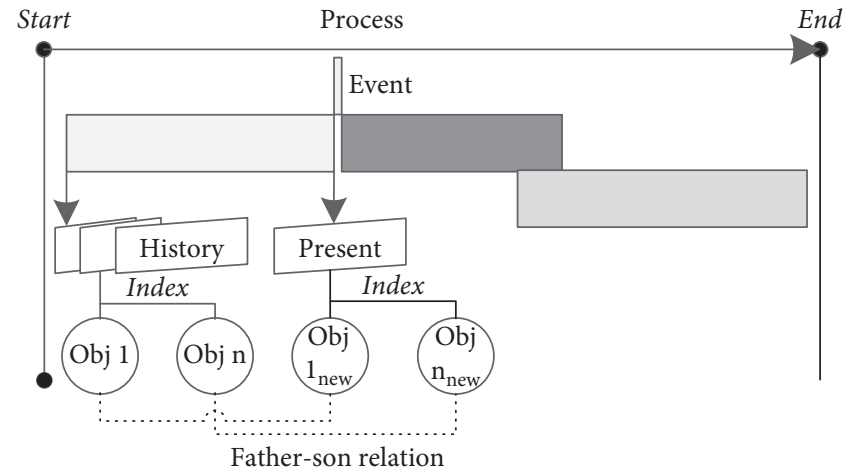

Record the changing status of objects before and after and the relationship between objects

A process-based dynamic spatiotemporal data model [13]

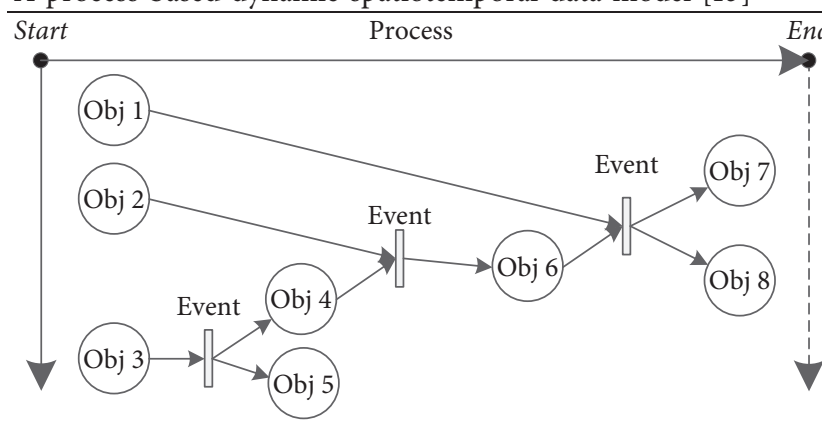

Research on spatiotemporal data model based on Petri net [14]

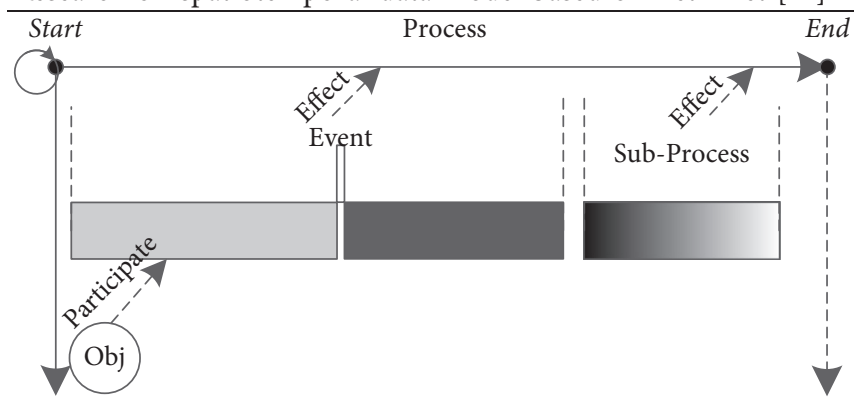

The graph model used to record and express the spatiotemporal changes with complex composition structure is effectively expressed, and the evolution process of objectevent-object is recorded

Spatiotemporal data model based on object-event-process and its application [15]

Database model based on spatiotemporal ontology [16]

enhancement/weakening with event ontology and process ontology when ontology relations are a concern. Events act on processes, with triggering, promoting, hindering, ending, and other types of relations. Process reacts to events with birth and end of events.
(2) Enriching the process of a temporal hierarchy method.

Based on the two-layer structure of both event and process, the method contributes to the temporal and spatial granularity of change semantics of the spatiotemporal data models through both subdivision and 
TABLE 3: Description of the enriching process of the temporal hierarchy method.

\begin{tabular}{lcc}
\hline Model diagram & & Model characteristics \\
\hline Start & Process & End
\end{tabular}

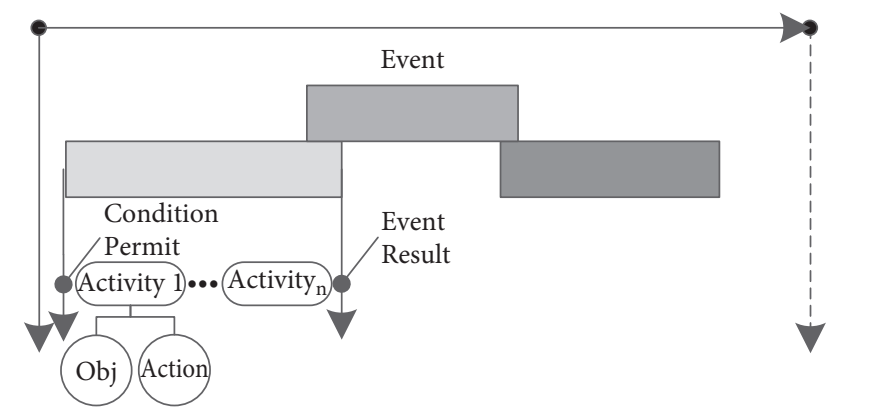

Space-time phenomena are expressed by activity, event, and process structure, and activity records objects and their actions

Toward knowledge discovery about geographic dynamics in spatiotemporal databases [18]

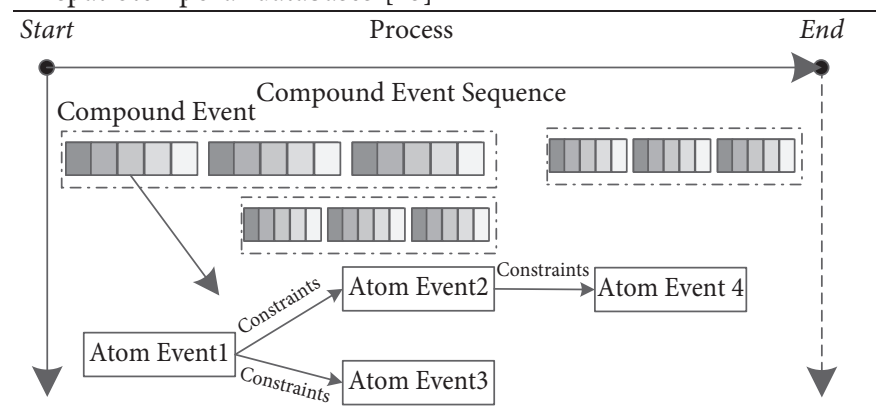

The expression of linkage complex events is achieved by introducing constraint relations into atomic events at the lowest level of spatiotemporal change semantics

A trapezoidal hierarchical description framework for spatiotemporal processes and its modeling example [19]

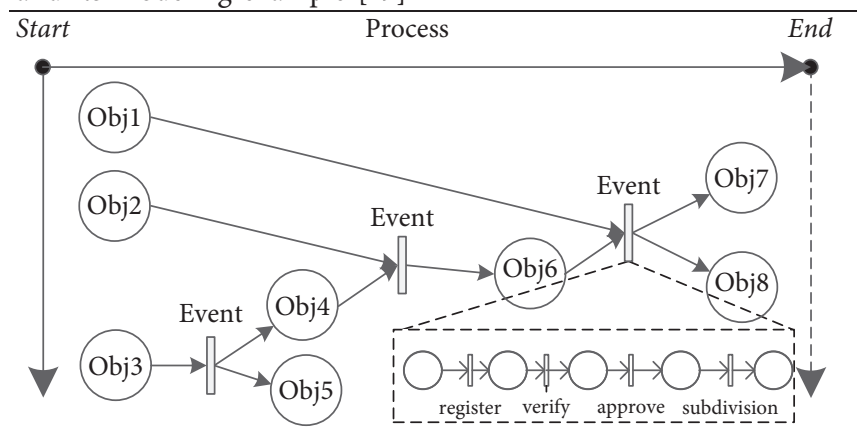

Research on spatiotemporal data model based on Petri net [14]

aggregation to meet the requirements of semantic expression of complex temporal and spatial phenomena (Table 3).

The knowledge extraction framework of a spatiotemporal dynamic system [18] uses "activities" smaller than event granularity to express object behavior. Events record the conditions of change, participating objects and results, and process organization events. Events also express dynamic system states and stages. The three-level spatiotemporal structure composed of activity-event-process can effectively resolve the key problems related to the calculation of a spatial relation and the discovery of an attribute change for knowledge mining of spatiotemporal dynamic systems. Spatiotemporal process using the trapezoid multilevel description framework (STP-TRAP) [19] divides the spatiotemporal change into four levels: atom event, evolution, evolution sequence, and spatiotemporal process. The granularity of the atom event is the smallest, which represents the instances of the constraint condition and corresponding spatiotemporal changes of atomic events.

The combination of atom events constitutes evolution (compound events). The evolution sequence organizes an evolution according to the temporal order and represents the compound spatiotemporal change with a meaning of a certain semantic. The spatiotemporal process is the top-level hierarchical description unit, which expresses the semantics of the whole spatiotemporal process. By organizing four levels from top to bottom, the spatiotemporal process is explicitly modeled, and the overall change process and the constraint relationship of the microscopic change are expressed. The transformation hierarchy of the Petri netbased spatiotemporal data model is extended, and the subprocess is introduced to express the process and trigger mechanism of the transformation process. This explicit expression of the spatiotemporal process has richer semantics and improves the efficiency of the analysis [20]. 
2.2.2. Homogenization Process. The homogenization process refers to the process type of the same internal change and the change of the semantic generally smaller than the event. It is often used to express the spatiotemporal process with continuous gradual change as a component of significant change- "event."

The most typical is the simulation of wildfires, storms, and other dynamic geographical phenomena, which are difficult to simulate exactly by functions due to various internal mechanisms and the characteristics of continuous gradual change in appearance. To express the semantics of the continuous gradual change, the observed sequence states are organized into "processes" according to specific semantics [21]. For the spatiotemporal evolution expression of entities such as seafront and sea ice through both classification and abstraction of homogenous change types, stage objects such as generation, expansion, stability, weakening, and extinction are defined [22, 23]. Based on sequence states, the process semantics of stage objects are refined by using spatiotemporal evolution functions. The processes are abstracted into mathematical functions, and the combinatorial relations of the simultaneous or sequential occurrence of "process algebra" are introduced to effectively simulate the complex spatiotemporal changes occurring internally in parallel settings [11]. The semantics of continuous gradual process also need to consider nonlinear continuous processes [24]. The characteristics of the intrinsic homogenous process model are shown in Table 4.

2.3. Spatiotemporal Object Relations. Traditional GIS data modeling only focuses on spatial attribute and thematic attribute information, while dynamic objects composed of events and processes interact with geographical objects to maintain the continuous development of spatiotemporal change in a spatiotemporal setting. This interaction is the key to the complete semantic representation of spatiotemporal changes and is also the feature of the ESTDM. Based on the available research results, this manuscript discusses both causality and composition.

2.3.1. Causality. Causality and change are inseparable. Because of the different mechanisms of action, the expression of causality varies greatly in different times and spaces. Therefore, this manuscript mainly discusses the predicates of causality between dynamic and geographical objects, which can be divided into a trigger, terminate, cause, permit, and maintain [25]. Dynamic objects act on geographic objects to "cause" state-change events, "trigger" new states, and "terminate" the original states. The occurrence of dynamic objects depends on geographical objects. When the accumulation of geographical object states reaches a certain extent, the occurrence of dynamic objects is "allowed." The state is a sufficient but not a necessary condition for the occurrence of dynamic objects. A special causality is the "maintenance" relationship. For the homogenization process, its internal change semantics are consistent, and when the process continues to occur, it implies the "maintenance" relationship.

2.3.2. Composition. The process and event of a dynamic object have a composition relation due to the difference of a granularity semantic of a spatiotemporal change. Based on the previous analysis, a structured process usually expresses the semantics of a large-scale change and consists of a series of events organized in sequence. Intrinsically, homogenous processes express continuous, progressive, and fine-grained change semantics and can be composed of semantically similar sequence states and spatiotemporal evolution functions. Therefore, the composition relations between spatiotemporal objects represent spatiotemporal change semantics from different levels, which can effectively improve the efficiency of spatiotemporal information retrieval of the model in practical applications.

The relationships among the three aspects are presented in Figure 1.

\section{Application Research of the ESTDM}

3.1. The Management of Cadastral Information. In the applications of both land and real estate, a change of spatiotemporal objects is mainly characterized by discrete time-varying characteristics [26]. The time-varying semantics of sequences such as the change of plot scope and utilization type can be expressed through the sequence of geographical events. In addition to the expression of sequential time-varying semantics, the recording and expression of evolutionary relationships between cadastral objects are also concerned in practical applications. The spatiotemporal change table is designed based on the invariance of an object identification [3] and graph theory [17]. Petri net [14] is used to explicitly record the spatiotemporal topological relationship between entities to realize the expression of the parent-child relationship of evolution relationship. To express a change of linkage relations of objects in evolutionary relations, the identification of the cadastral object that changes simultaneously with other cadastral objects is added to the concept of events [27], and the updated constraint rules of the cadastral object are formulated based on the causality and linkage rules among cadastral objects, processes, and events [26]. In terms of the expression of the business process related to a land change, the combination of "atomic event" and "event operator" constitutes the "composite event" of land allocation, which flexibly meets the change of the business demand for the land allocation [28, 29]. The business processes of the land allocation are expressed through hierarchical expansion of the spatiotemporal data model based on the Petri net [20]. While "process" is defined as a change of a cadastral attribute, an "event" is defined as a land registration event, that is, external cause of cadastral change [24], which is organically connected to the change of the cadastral use concerning the land allocation business. 
TABle 4: Description of intrinsic homogenous spatiotemporal processes.

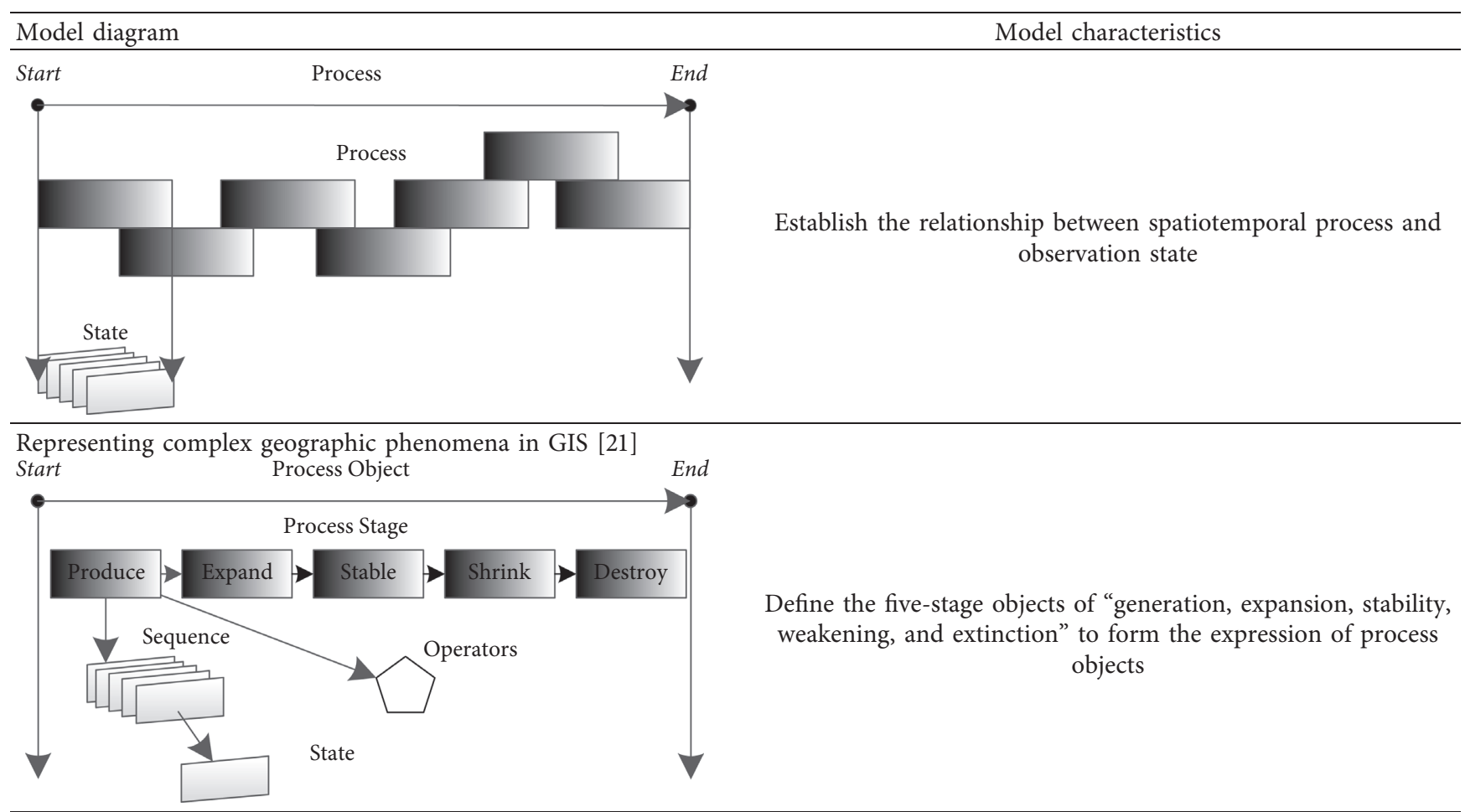

Research on marine spatiotemporal process data model and its prototype system construction [22]

Research on process-oriented spatiotemporal data model [23]

Start Event End

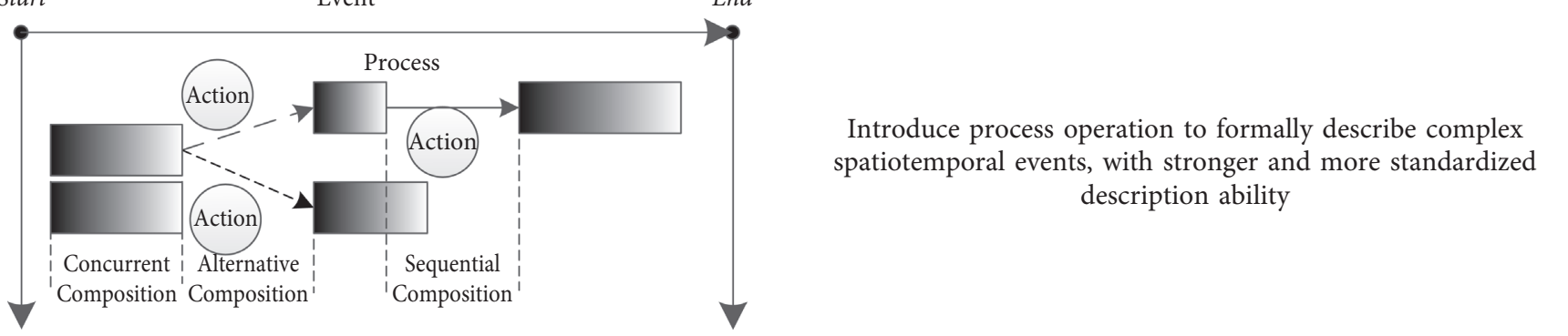

Event-oriented approaches to geographic phenomena [11]

Start Event (Externally Driven) End

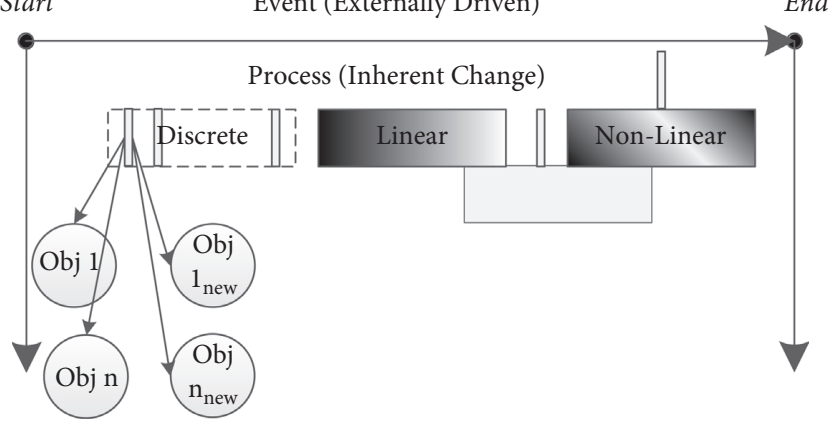

Propose three types of processes: discrete, linear, and nonlinear

Research on an improved temporal model based on event-process [24]

3.2. Re-Enactment and Reasoning of Physical Geographic Phenomena. The evolution of the physical geographic phenomenon is the result of various factors influencing each other and energy transfer, which has the characteristics of continuous gradual change and obvious causal relationships. In the process of modeling, it is necessary to make a lot of improvements to the ESTDM, which mainly focuses on the simulation of the evolution process and the coherence expression of spatiotemporal change semantics. 


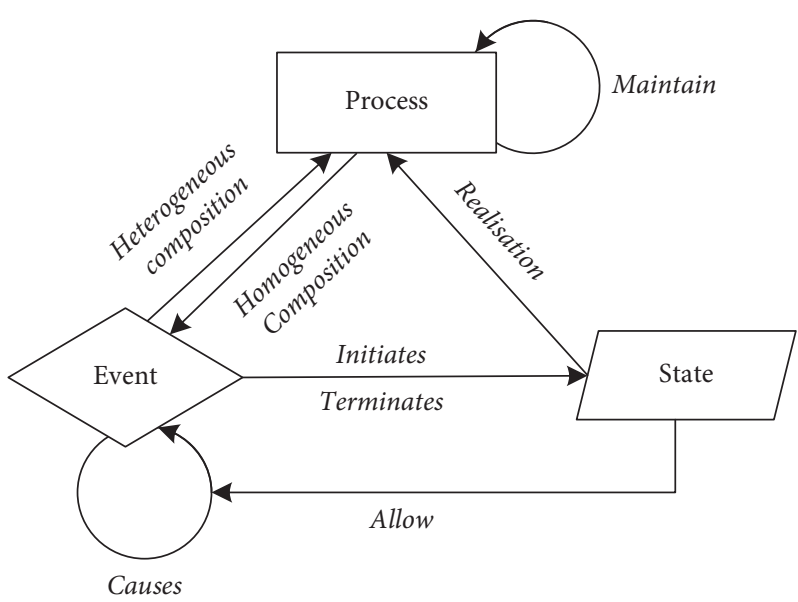

Figure 1: Relationships between events, processes, and states.

A sea ice ontology concerning sea ice objects, events, and processes was constructed utilizing ice caps, icebergs, and floating ice which are called sea ice objects, and events such as "falling off" of ice caps and "drift" of icebergs, as well as processes such called as "formation," "rupture," "drift," and "agglomeration" of floating ice. The relationships such as "instance," "composition," "action" and "trigger," the changes and causes of sea ice are effectively expressed [16]. Based on the idea of a continuous and progressive change of spatiotemporal process expression $[30,31]$, the objects of the ocean vortex process with complete evolution characteristics are classified and selected. The inference responses to comprehensive spatiotemporal query questions are realized through the decomposition of the temporal conditional statement, process object integration, and the processes of the sequence object integration [23]. Based on Region Connection Calculus (RCC) and Temporal Logic theory, the RCC Association Event (RAE) model, associated with the topological time-varying of "regional object" and "event," is proposed, and the path law of tropical cyclone is effectively mined by using the probability model [32]. Some of the recent researches dealing with different disciplines can be found [33-36].

\section{Findings}

The ESTDM is a dynamic spatiotemporal information modeling method, which can effectively simulate spatiotemporal changes and reflect the intrinsic laws of changes by modeling spatiotemporal information, changes, and object relations when temporal and spatial changes need to be modeled.

This manuscript expounds on the modeling theory of the ESTDM, compares the characteristics of geographical and spatiotemporal dynamic objects, studies the concepts and modeling methods of structuration and homogenization processes, and discusses the causal and composition relationships between spatiotemporal objects.

The characteristics of both discrete and continuous change models are analyzed based on the management of the cadastral object, the re-enactment of a physical geographic phenomenon, and reasoning. It is found that the ESTDM is an effective method to deal with dynamic spatiotemporal information by modeling spatiotemporal information, changes, and object relations, which can effectively simulate spatiotemporal change and reflect the intrinsic laws of change. However, there are still several challenges due to the dynamics and complexity of space-time phenomena and the characteristics of modeling objects in various fields.

4.1. Research and Understanding of the Spatiotemporal Dynamic Semantics. The research of spatiotemporal objects has made some theoretical achievements, but it is still in its infancy. Spatiotemporal dynamic semantics are closely related to spatiotemporal granularity, which is instantiated in the mutual composition relation of events and processes, which would lead to spatiotemporal semantic crossover and data redundancy to a certain extent. A standard, clearer, and comprehensive dynamic semantic of a spatiotemporal expression model needs to be introduced.

4.2. The Knowledge Mining of the Spatiotemporal Change. The ESTDM resolves the problems of spatiotemporal data organization and expression of spatiotemporal evolution relations and can better serve the application areas when the management of the cadastral information and the re-enactment of the physical geographic phenomenon is a concern. Based on solving the problem of dynamic spatiotemporal data modeling, it is necessary to continuously introduce key technologies such as probabilistic and automatic inference machine models to realize the promotion of data management to knowledge service.

4.3. Intelligent Aggregation of the Multisourcel MultivariantData. With the continuous development of emerging communication technologies such as 5G, the Internet of Things (IoT), and sensor networks, "digital twin cities" are no longer far away. Available models mainly focus on simulating the semantic changes of a single type of 
geographical object or natural geographic phenomenon. Therefore, for the expression of complex spatiotemporal changes involving multisource/multivariant data and interaction of multitype spatiotemporal objects, continuous exploration is still needed.

\section{Conclusion}

In this manuscript, we study the event-based spatiotemporal data modeling theory based on three aspects of the cognitive theory, which are the spatiotemporal object, the concept of the spatiotemporal dynamic object, and the spatiotemporal object relationship. Then, the implementation characteristics of the models were analyzed regarding the management of cadastral information, analog natural disaster phenomena, and reasoning. Finally, the key points and difficulties of an event-based spatiotemporal data modeling and prospective developmental trends were discussed to provide insights with spatiotemporal data modeling.

\section{Data Availability}

All the data are included in the manuscript.

\section{Conflicts of Interest}

The authors declare that they have no conflicts of interest.

\section{References}

[1] M. Yuan and J. Mcintosh, GIS Representation For Visualizing and Mining Geographic Dynamics, Transactions in GIS, vol. 3, no. 2, , pp. 3-10, 2003.

[2] J. Gong, X. Li, and H. Wu, "Real-time GIS spatiotemporal data model," Journal of Geodesy and Geoinformation Science, vol. 14, no. 3, pp. 226-232, 2014.

[3] Z. Xu, F. Bian, and J. Chen, "Temporal GIS model based on event semantics," Journal of Wuhan University (Natural Science Edition), vol. 27, no. 3, pp. 311-315, 2002.

[4] L. Meng, C. Zhao, Z. Lin, and C. Huang, "Research and implementation of spatiotemporal data model based on the time-varying sequence of geographical events," Journal of Wuhan University (Natural Science Edition), vol. 28, no. 2, pp. 202-207, 2003.

[5] K. E. Grossner, "Event objects for spatial history," in Proceedings of the Sixth International Conference on Geographic Information Science, Zurich, Switzerland, September 2010.

[6] D. J. Peuquet and N. Duan, "An event-based spatiotemporal data model (ESTDM) for temporal analysis of geographical data," International Journal of Geographical Information Systems, vol. 9, no. 1, pp. 7-24, 1995.

[7] A. Galton, "Fields and objects in space, time, and space-time," Spatial Cognition and Computation, vol. 4, no. 1, pp. 39-68, 2004.

[8] A. Galton, "Experience and history: processes and their relation to events," Journal of Logic and Computation, vol. 18, no. 3, pp. 323-340, 2008.

[9] A. Galton and R. Mizoguchi, "The water falls but the waterfall does not fall: new perspectives on objects, processes and events," Applied Ontology, vol. 4, no. 2, pp. 71-107, 2009.
[10] G. Pierre and S. Barry, "SNAP and SPAN: towards dynamic spatial ontology," Spatial Cognition and Computation, vol. 4, no. 1, pp. 69-104, 2004.

[11] M. Worboys, "Event-oriented approaches to geographic phenomena," International Journal of Geographical Information Science, vol. 19, no. 1, pp. 1-28, 2005.

[12] A. Galton, "On what goes on: the ontology of processes and events: conference on formal ontology in information systems," in Proceedings of the Fourth International Conference, Baltimore, MD, USA, November 2006.

[13] F. Zhang, R. Liu, N. Liu et al., "A process-based dynamic spatiotemporal data model," Journal of Sun Yat-sen University (Social Science Edition), vol. 47, no. 2, pp. 123-126, 2008.

[14] Z. Yin and L. Li, "Research on spatiotemporal data model based on Petri net," Journal of Wuhan University (Natural Science Edition), vol. 29, no. 8, pp. 740-743, 2004.

[15] X. Chen, L. Li, and M. Li, "Spatiotemporal data model based on object event process and its application," Geography and Geo-Information Science, vol. 29, no. 3, pp. 14-20, 2013.

[16] X. Chen and J. Zhu, "Database model based on spatiotemporal ontology," Geography and Geo-Information Science, vol. 26, no. 5, pp. 1-6, 2010.

[17] Z. C. Yin, L. Li, and Z. Ai, "Research on spatiotemporal data model based on graph theory," Journal of Geodesy and Geoinformation Science, vol. 32, no. 2, pp. 168-172, 2003.

[18] Y. May, "Toward knowledge discovery about geographic dynamics in spatiotemporal databases," in Geographic Data Mining and Knowledge Discovery, pp. 347-365, , CRC Press, Boca Raton, FL, USA, 2009, 2nd edn.

[19] J. Xie, R. Liu, N. Liu et al., “A Trapezoidal hierarchical description framework for spatiotemporal processes and its modeling example," Journal of Geodesy and Geoinformation Science, vol. 36, no. 3, pp. 321-328, 2007.

[20] Z. Huang and X. Feng, "Spatiotemporal process modeling of land change based on Petri net," Journal of Geodesy and Geoinformation Science, vol. 34, no. 3, pp. 239-245, 2005.

[21] Y. May, "Representing complex geographic phenomena in GIS," The American Cartographer, vol. 28, no. 2, pp. 83-96, 2001.

[22] C. Xue and Q. Dong, "Research on marine spatiotemporal process data model and its prototype system construction," Marine Science Bulletin, vol. 31, no. 6, pp. 667-674, 2012.

[23] C. Xue, C. Zhou, F. Su, Q. Dong, and J. Xie, "Research on process-oriented spatiotemporal data model," Journal of Geodesy and Geoinformation Science, vol. 39, no. 1, pp. 95$101,2010$.

[24] C. Wu and G. Lu, "Research on an improved temporal model based on event-process," Journal of Wuhan University (Natural Science Edition): Information Science Edition, vol. 33, no. 12 , pp. 1250-1253, 2008.

[25] E. Allen, G. Edwards, and Y. Bédard, "Qualitative Causal Modeling in Temporal GIS," in Proceedings of the International Conference on Spatial Information Theory, Semmering, Austria, September 1995.

[26] F. Zhang, N. Liu, R. Liu et al., "Research on object-oriented cadastral spatiotemporal process expression and data update model," Journal of Geodesy and Geoinformation Science, vol. 39, no. 3, pp. 303-309, 2010.

[27] L. Meng, P. Chen, X. Tong et al., "Research on the spatiotemporal data model of cadastral objects based on the whole life cycle and improvement events," Geomatics World, vol. 22, no. 4, pp. 51-56, 2015.

[28] C. J. Jiang, J. Chen, and J. Jiang, "Event-based Spatio-temporal database design," Acta Geodaetica et Cartographica Sinica, vol. 29, no. 1, pp. 64-70, 2000. 
[29] J. Chen and J. Jiang, "An event-based approach to spatiotemporal data modeling in land subdivision systems," Geoinformatics, vol. 4, no. 4, pp. 387-402, 2000.

[30] C. Claramunt, C. Parent, and M. Thériault, "Design pstemporal processes," Data Mining and Reverse Engineering, pp. $455-475,1998$.

[31] F. Su and C. Zhou, "Framework foundation and prototype construction of process geographic information system," Geographical Research, vol. 25, no. 3, pp. 477-484, 2006.

[32] X. Chen, Research on the Spatiotemporal Data Model and Time-Varying Analysis Model of Events and Processes, Central South University, Changsha, China, 2011.

[33] Ş. Boghiu and D. Gîfu, "A spatial-temporal model for event detection in social media," Procedia Computer Science, vol. 176, pp. 541-550, 2020.

[34] W. Xiong, H. Chen, N. Guo et al., ENSTDM: An ENtity-Based Spatial-Temporal Data Model and Case Study in Real Estate Management, Spatial Data and Intelligence, Springer, Heidelberg, Germany, 2021.

[35] M. Yu, "A graph-based spatiotemporal data framework for 4D natural phenomena representation and quantification-an example of dust events," Int. J. Geo-Information, vol. 9, no. 127, pp. 1-17, 2020.

[36] M. Yu, M. Bambacus, G. Cervone et al., "Spatiotemporal event detection: a review," International Journal of Digital Earth, vol. 13, 2020. 